\title{
The small FNR regulon of Neisseria gonorrhoeae: comparison with the larger Escherichia coli FNR regulon and interaction with the NarQ-NarP regulon
}

\author{
Rebekah N Whitehead $^{\dagger 1}$, Tim W Overton ${ }^{\dagger 1}$, Lori AS Snyder ${ }^{2}$, \\ Simon J McGowan ${ }^{3}$, Harry Smith ${ }^{4}$, Jeff A Cole*1 and Nigel J Saunders*2
}

Address: ${ }^{1}$ School of Biosciences, University of Birmingham, Edgbaston, Birmingham B15 2TT, UK, ${ }^{2}$ The Bacterial Pathogenesis and Functional Genomics Group, The Sir William Dunn School of Pathology, University of Oxford, South Parks Road, Oxford, OX1 3RE, UK, ${ }^{3}$ The Computational Biology Research Group, The Sir William Dunn School of Pathology, University of Oxford, South Parks Road, Oxford, OX1 3RE, UK and ${ }^{4}$ The Medical School, The University of Birmingham, Edgbaston, Birmingham B15 2TT, UK

Email: Rebekah N Whitehead - RNW283@bham.ac.uk; Tim W Overton - t.w.overton@bham.ac.uk; Lori AS Snyder - lori.snyder@path.ox.ac.uk; Simon J McGowan - simon.mcgowan@ path.ox.ac.uk; Harry Smith - hsmithcbefrsbham@btopenworld.com; Jeff A Cole* - j.a.cole@bham.ac.uk; Nigel J Saunders* - nigel.saunders@path.ox.ac.uk

* Corresponding authors †Equal contributors

Published: 29 January 2007

BMC Genomics 2007, 8:35 doi:10.1/86/147|-2/64-8-35
Received: 7 September 2006

Accepted: 29 January 2007

This article is available from: http://www.biomedcentral.com/I47/-2/64/8/35

(c) 2007 Whitehead et al; licensee BioMed Central Ltd.

This is an Open Access article distributed under the terms of the Creative Commons Attribution License (http://creativecommons.org/licenses/by/2.0), which permits unrestricted use, distribution, and reproduction in any medium, provided the original work is properly cited.

\begin{abstract}
Background: Neisseria gonorrhoeae can survive during oxygen starvation by reducing nitrite to nitrous oxide catalysed by the nitrite and nitric oxide reductases, AniA and NorB. The oxygen-sensing transcription factor, FNR, is essential for transcription activation at the aniA promoter, and full activation also requires the twocomponent regulatory system, NarQ-NarP, and the presence of nitrite. The only other gene known to be activated by the gonococcal FNR is ccp encoding a cytochrome $c$ peroxidase, and no FNR-repressed genes have been reported in the gonococcus. In contrast, FNR acts as both an activator and repressor involved in the control of more than 100 operons in $E$. coli regulating major changes in the adaptation from aerobic to anaerobic conditions. In this study we have performed a microarray-led investigation of the FNR-mediated responses in N. gonorrhoeae to determine the physiological similarities and differences in the role of FNR in cellular regulation in this species.
\end{abstract}

Results: Microarray experiments show that $N$. gonorrhoeae FNR controls a much smaller regulon than its $E$. coli counterpart; it activates transcription of aniA and thirteen other genes, and represses transcription of six genes that include $d n r N$ and norB. Having previously shown that a single amino acid substitution is sufficient to enable the gonococcal FNR to complement an E. coli fnr mutation, we investigated whether the gonococcal NarQ-NarP can substitute for $E$. coli NarX-NarL or NarQ-NarP. A plasmid expressing gonococcal narQ-narP was unable to complement $E$. coli narQP or narXL mutants, and was insensitive to nitrate or nitrite. Mutations that progressively changed the periplasmic nitrate sensing region, the $\mathrm{P}$ box, of $E$. coli NarQ to the sequence of the corresponding region of gonococcal NarQ resulted in loss of transcription activation in response to the availability of either nitrate or nitrite. However, the previously reported ligand-insensitive ability of gonococcal NarQ, the "locked on" phenotype, to activate either E. coli NarL or NarP was confirmed.

Conclusion: Despite the sequence similarities between transcription activators of $E$. coli and N. gonorrhoeae, these results emphasise the fundamental differences in transcription regulation between these two types of pathogenic bacteria. 


\section{Background}

Neisseria gonorrhoeae is an obligate human pathogen with no known environmental reservoirs. It can be isolated from gonorrhoea patients in clinical samples in which obligately anaerobic bacteria are abundant [1,2], suggesting that gonococci encounter and survive without oxygen in their natural habitat. Clark and her colleagues have shown that gonococci can grow anaerobically using a truncated denitrification pathway in which nitrite is reduced to nitrous oxide, catalysed by the copper-containing nitrite reductase, AniA, and the single subunit nitric oxide reductase, NorB [2-5]. Nitrite reduction is severely repressed by oxygen, but is induced during anaerobic growth by the global transcription factor, FNR (for regulator of fumarate and nitrate reduction), and by a two-component regulatory system that we designated NarQ-NarP $[5,6])$.

Until complete genome sequences became available in the last ten years, it was commonly assumed that obligate pathogens rely less on transcription control than more versatile bacteria that occupy a variety of niches outside of their mammalian hosts. With reference to N. gonorrhoeae, this impression was reinforced by the fact that $\operatorname{ccp}$ (encoding a cytochrome $c$ peroxidase $[5,7])$ is the only gene other than aniA that is known to be regulated by the gonococcal FNR, and by the widespread distribution of repeat DNA sequences that promote a high frequency of genetic variation-based expression control resulting in gene silencing, phase variation, and antigenic shift. The availability of the complete genome sequence and pan-Neisseria microarrays provide an opportunity to test these assumptions directly by comparing the extent of the $N$. gonorrhoeae FNR regulon with that of the recently-published $E$. coli FNR regulon [8]. As full expression of aniA in the gonococcus and both of the major nitrite reductases in E. coli all require a functional two-component regulatory system (NarQP in the gonococcus; both NarQP and NarXL in E. coli), we have also investigated the similarities and differences between the NarQP systems of these bacteria.

\section{Results \\ Microarray analysis of the gonococcal FNR regulon}

To determine the range of functions regulated by FNR in $N$. gonorrhoeae, the $\mathrm{fnr}^{+}$parental strain, RUG7001, and its isogenic fnr mutant, RUG7022, were grown in poorly aerated cultures until oxygen became limiting and aniA expression had been induced (as indicated by the disappearance of nitrite from the medium). The $f n r^{+}$parental strain grew exponentially until the nitrite had been reduced. In contrast, the fnr mutant grew at a constant rather than an exponential rate that was similar to the oxygen-limited growth of the parental strain in the absence of nitrite (Fig. 1). To distinguish between genes differentially expressed in response to growth rate from those regulated directly by FNR in response to oxygen limitation, RNA was extracted from the $\mathrm{fnr}^{+}$parental strain grown in the presence or absence of nitrite, and from the fnr mutant grown in the presence of nitrite. These RNA preparations were analysed using two pair wise comparisons: $\mathrm{fnr}^{+}$gonococci growing exponentially in the presence of nitrite compared to the fnr mutant; and $f n r^{+}$gonococci growing in the absence of nitrite compared to the fnr mutant. Data were analysed according to fold-change ( $>2$-fold difference between the two strains) and a Student's t-test, using a cut-off $p$ value of 0.01 , reflecting the fact that six biological replicates were used in this experiment. Fourteen transcripts were more abundant in the parental strain than the fnr mutant, while six were more abundant in the mutant, suggesting FNR repression (Table 1). Five of the transcripts were more abundant in the $\mathrm{fnr}^{+}$strain during growth in both the presence and absence of nitrite, suggesting direct FNR regulation. The most up-regulated transcripts encode a short transcript of unknown function (46.3-fold, $p 1.1 \times 10^{-5}$ with nitrite; 73.06 -fold, $p 7.3 \times 10^{-}$ ${ }^{6}$ without nitrite), and a putative iron uptake outer membrane protein designated OmpU (NGO1688; 12.27-fold, p $3.2 \times 10^{-4}$ with nitrite; 6.6-fold, p $7.3 \times 10^{-5}$ without nitrite) [[9]; see also comments in the Entrez nucleotide entry for $N$. meningitidis ompU, accession AF118122]. Also activated were the nitrite reductase gene, aniA (6.02-fold, p $1.9 \times 10^{-5}$ with nitrite; 3.56 -fold, p $8.2 \times 10^{-4}$ without nitrite), NGO1215 encoding a highly conserved hypothetical protein (4.57-fold, $p 4.8 \times 10^{-4}$ with nitrite; 3.52 fold, $p 3.2 \times 10^{-5}$ without nitrite); and NGO0546 encoding the Res subunit of a Type III restriction-modification system similar to the EcoPI enzyme (2.31-fold, p $2.1 \times 10^{-3}$ with nitrite; 2.88 -fold, $p 5.2 \times 10^{-5}$ without nitrite). Nine further transcripts were more highly expressed in the $\mathrm{fnr}^{+}$ strain compared to the fnr mutant, but only during growth in the presence of nitrite. These transcripts are possibly regulated in response to growth rate rather than by FNR. Only one of these genes, NGO0602, encoding a putative MerR-family transcription regulator, was also more highly expressed in the $\mathrm{fnr}^{+}$strain grown in the absence of nitrite (2.8-fold), but the regulation of this gene was not as statistically significant ( $p$ 0.05).

Most down-regulated by FNR was the NGO1716 transcript encoding a putative phosphotransferase (COG3178; 0.14-fold, p $9.6 \times 10^{-6}$ with nitrite; 0.18-fold, p $8.1 \times 10^{-6}$ without nitrite), followed by two genes implicated in nitric oxide metabolism, $\operatorname{dnrN}$ (0.37-fold, $p 0.12$ with nitrite; 0.19 -fold, $p 8.4 \times 10^{-6}$ without nitrite), and norB (0.6-fold, $p 0.84$ with nitrite; 0.28 -fold, $p 2.9 \times 10^{-3}$ without nitrite). Two transcripts were less abundant in the parental strain during growth in the presence of nitrite: cysteine synthetase (cysK gene; NGO0340; 0.29-fold, $p 7.3$ $\times 10^{-3}$ ); and $g \ln Q$ (NGO0374) encoding a component of an ABC-type amino acid transporter (0.47-fold; $p 3.4 \times 10^{-}$ 


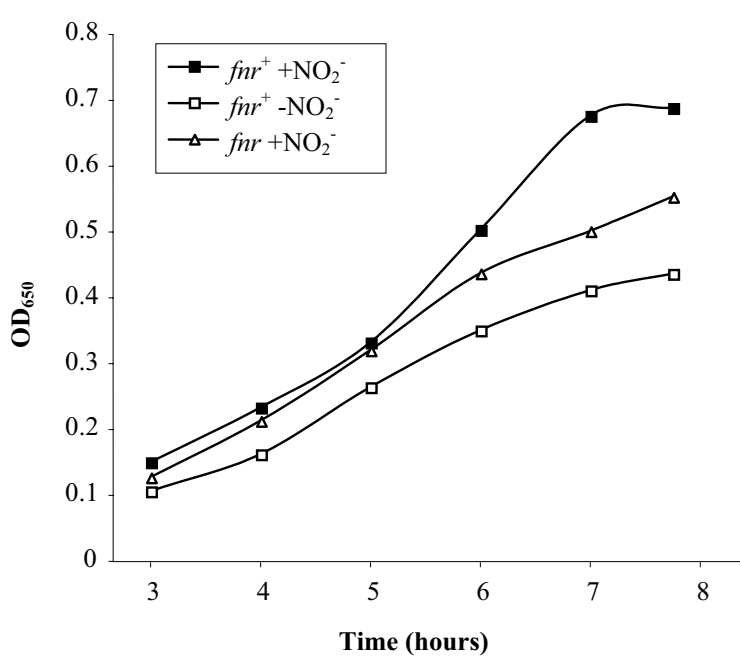

Figure I

Growth characteristics of strain RUG700I ( $\left.f n r^{+}\right)$in the presence and absence of $5 \mathrm{mM}$ sodium nitrite and strain RUG7002 ( $f n r)$ in the presence of nitrite. Since expression of the nitrite reductase AniA is dependent upon FNR, the fnr strain cannot respire nitrite.

$\left.{ }^{4}\right)$. The transcript for a Neisseria-specific protein encoded by NGO1428 was more abundant in the mutant only in the absence of nitrite $\left(0.49\right.$-fold, $\left.p 2.6 \times 10^{-4}\right)$. These data are summarized in Table 1.

The results of the microarray experiments can be interactively interrogated in an on-line graphical GBrowse database at http://tinyurl.com/fu2um, where the fold ratio, number of observations for each gene, Student's t-test, Cyber-T p-values, XNG and NGO annotations, and the microarray probe locations can be visualized, and searched using chromosomal locations, gene names, or gene identifiers. The results from each experiment can be viewed individually or in combination to compare the results, and users can add their own local annotations. The results of these experiments can also be seen in direct comparison with the expression data obtained in a previous microarray study addressing expression associated with narPQ [10].

\section{Bioinformatic and ChIP analysis of promoters of genes differentially expressed in the fnr mutant}

Putative FNR binding sites with at least a 7/10 match to the E. coli consensus, TTGATNNNNATCAA, were identified within 200 base pairs upstream of the translation start codon of eleven of the genes revealed by the microarray experiments to be differentially expressed in the $f n r$ mutant and its parent (Table 1). A strain containing a chromosomal fnr-3xFLAG fusion was constructed (N. gonorrhoeae strain JCGC502), grown microaerobically both in the presence and absence of nitrite to the late exponential phase, DNA-binding proteins were cross linked to the chromosome, the bacteria were lysed and chromosomal DNA was sheared. Anti-FLAG antibodies were used to immunoprecipitate FNR-DNA complexes, which were decrosslinked and the DNA released was purified. The quantity of each promoter fragment in the immunoprecipitated DNA pool was measured by realtime PCR, relative to the FNR-independent $h p t$ promoter. Promoter fragments enriched $60 \%$ or more in at least two independent experiments scored positive (Table 1).

Only one of the fourteen genes potentially activated by FNR had previously been reported to be FNR-dependent: aniA, encoding a nitrite reductase $[5,6]$. Consistent with the microarray data reported above, FNR binding to $\mathrm{P}_{\text {anis }}$ was confirmed by ChIP, providing a positive control for the ChIP data. The ChIP experiments also confirmed FNRbinding to the promoter regions of the most highly FNRactivated transcript (NMB1205) and ompU. Although the ompU promoter has no recognisable FNR binding site, multiple potential half-sites are present in the promoter region. Conversely, even though open reading frames NGO1215 and NGO0546 have potential FNR binding sites upstream, FNR binding was not detected by ChIP. This emphasises that caution is required when drawing conclusions from data based upon either of these techniques alone.

\section{Proteomic analysis of the FNR regulon}

A study of protein expression in $f n r^{+}$and $f n r$ gonococci revealed fewer differences than were identified in the microarray study. Comparison of the cytoplasmic proteins of the $f n r^{+}$parental strain, RUG7001, and the $f n r$ mutant, strain RUG7022, grown in the presence of nitrite revealed no significant differences in protein expression (data not shown). In contrast, three membrane proteins were more abundant in the $\mathrm{fnr}^{+}$strain: nitrite reductase, AniA; nitric oxide reductase, NorB; and the septum sitedetermining cell division protein, MinD [11]. Although norB transcription is independent of FNR [12], more NorB accumulated during nitrite reduction by the $\mathrm{fnr}^{+}$strain than in the $f n r$ mutant. The explanation for this apparent contradiction is that transcription from the nor B promoter is induced by NO $[9,12]$, so because AniA is not synthesised in the mutant, no NO would be generated to activate the expression of nor $B$. As the microarray data did not identify $\min D$ expression to be activated by FNR, the apparent differential expression of MinD protein, revealed by proteomic analysis, is more likely to be due to a growth rate effect than to a direct effect of FNR. 
Table I: Genes differentially expressed in $\mathrm{fnr}^{+}$and $\mathrm{fnr}$ strains of $\mathrm{N}$. gonorrhoeae.

$f n r^{+}+\mathrm{NO}^{2-l} / \mathrm{fnr} \quad f n r^{+}-\mathrm{NO}^{2-l} / f n r \quad$ Gene number Gene name

\section{Gene number Gene name}

Product

FNR binding site upstream

Sequence

Location

Transcripts more abundant in the Ratio t-test Ratio t-test fnr ${ }^{+}$strain

\begin{tabular}{|c|c|c|c|c|c|c|c|c|c|}
\hline 46.30 & $1.1 \times 10 p$ value $^{-5}$ & 73.06 & $7.3 \times 10 p$ value $^{-6}$ & NMBI 205 & & Transcript of unknown function & TTGATTTGAATCAA & 41.5 & Yes \\
\hline 12.27 & $3.2 \times 10^{-4}$ & 6.60 & $7.3 \times 10^{-5}$ & NGOI688 & ompU & Putative iron uptake OMP & none & & Yes \\
\hline 6.02 & $1.9 \times 10^{-5}$ & 3.56 & $8.2 \times 10^{-4}$ & NGOI276 & aniA & Nitrite reductase & TTGACTTAAATTAA & 88.5 & Yes $\S$ \\
\hline 4.57 & $4.8 \times 10^{-4}$ & 3.52 & $3.2 \times 10^{-5}$ & NGOI2I5 & & Conserved hypothetical protein (COG2847) & TTAATCTACATCAA & 73.5 & NE \\
\hline 2.31 & $2.1 \times 10^{-3}$ & 2.88 & $5.2 \times 10^{-5}$ & NGO0546 & res & $\begin{array}{c}\text { Type III restriction-modification system EcoPI } \\
\text { enzyme }\end{array}$ & TTGAATGTGATTAT & 106.5 & NE \\
\hline 4.21 & $1.9 \times 10^{-3}$ & ND & & XNGI30I & $\operatorname{nos} R$ & Regulatory protein & TTGATTTGCATCAA & 20.5 & NT \\
\hline 2.85 & $3.1 \times 10^{-3}$ & 0.72 & 0.06 & NGO162I & & Putative phage associated protein & none & & NT \\
\hline 2.75 & $1.0 \times 10^{-3}$ & 0.83 & 0.28 & NGOI622 & & Putative phage associated protein & none & & NT \\
\hline 2.70 & $4.1 \times 10^{-3}$ & 0.86 & 0.47 & NGO0472 & & Putative phage associated protein & none & & NT \\
\hline 2.63 & $1.7 \times 10^{-4}$ & 1.00 & 0.51 & NGOI455 & & $\begin{array}{l}\text { Putative NRAMP family Manganese/Iron } \\
\text { transporter }\end{array}$ & none & & NE \\
\hline 2.56 & $5.1 \times 10^{-3}$ & 2.80 & 0.05 & NGO0602 & & Putative MerR family transcriptional regulator & TTGTTTCATTTCCA & 130.5 & NT \\
\hline 2.20 & $9.5 \times 10^{-3}$ & 1.21 & 0.16 & NGO0087 & & Hypothetical protein & none & & NT \\
\hline 2.10 & $2.0 \times 10^{-3}$ & 1.02 & 0.83 & NGO0473 & & Neisseria specific protein. & GTGATTCTGACAAA & 199.5 & NE \\
\hline 2.01 & $1.4 \times 10^{-3}$ & 1.00 & 0.71 & NGOI6I5 & & Putative phage associated protein & $\begin{array}{l}\text { TTCAGCTGCTTCAA } \\
\text { CTGATCAGCCTCAT }\end{array}$ & $\begin{array}{l}70.5 \\
25.5\end{array}$ & NT \\
\hline \multicolumn{10}{|c|}{ Transcripts more abundant in the fnr strain } \\
\hline 0.14 & $9.6 \times 10^{-6}$ & 0.18 & $8.1 \times 10^{-6}$ & NGO1716 & & Putative phosphotransferase & TTGAGTGAAAATAA & 85.5 & Yes \\
\hline 0.29 & $7.3 \times 10^{-3}$ & 1.23 & 0.39 & NGO0340 & cysK & Cysteine synthetase & none & & Yes \\
\hline 0.47 & $3.4 \times 10^{-4}$ & 1.47 & 0.04 & NGO0374 & $g \ln Q$ & Amino acid $A B C$ transporter & none & & NT \\
\hline 0.37 & 0.12 & 0.19 & $8.4 \times 10^{-6}$ & NGO0653 & $d n r N$ & Putative NO- response protein & ATGTTCAAAACCAA & 36.5 & NE \\
\hline 0.60 & 0.84 & 0.28 & $2.9 \times 10^{-3}$ & NGOI 275 & norB & Nitric oxide reductase & TGAATTTTAAACAA & 33.5 & Yes $\S$ \\
\hline 0.91 & 0.31 & 0.49 & $2.6 \times 10^{-4}$ & NGOI428 & & Short Neisseria specific protein & none & & NE \\
\hline
\end{tabular}

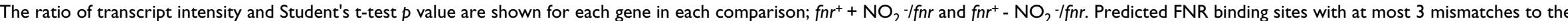

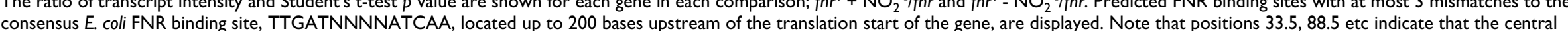

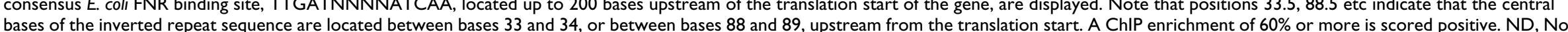

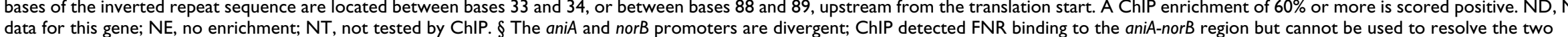




\section{Effects of iron deprivation and peroxide stress on an fnr mutant}

In light of the altered expression of the genes associated with iron transport (ompU and the putative NRAMP family member NGO1455) it was determined whether FNR is important in metal ion uptake. The ability of a gonococcal fnr mutant to survive metal ion limitation was tested. Microaerobically grown $f n r$ mutant and parent were treated with $200 \mu \mathrm{M}$ dipyridyl, a chelator of iron and manganese ions. Viable counts made at regular intervals for up to one hour showed there was no significant difference in survival between the $f n r^{+}$and $f n r$ gonococci.

Considering that genes predicted to be involved in defence against ROS (ccp [7]) or induced upon exposure to ROS (NGO1428 and NGO1716 [13]) were observed to be regulated by FNR, the ability of an fnr mutant to survive oxidative stress was tested. Oxygen-limited cultures of gonococci, both the fnr mutant and the parental strain, were subjected to $10 \mathrm{mM}$ hydrogen peroxide. Viable counts taken at intervals up to one hour showed, as with the metal chelation experiment, no significant difference in survival between the $f n r^{+}$and $f n r$ bacteria.

\section{Why cytochrome c peroxidase was not identified as being FNR-regulated by microarray analysis}

Expression of the cytochrome $c$ peroxidase is activated by FNR in response to oxygen [5]. Mature CCP protein was detected by staining gonococcal whole cell or membrane proteins separated by SDS-PAGE for haem-dependent peroxidase activity. However, in the present microarray study, FNR-dependent expression of the ccp gene was not observed because of the low level of expression of $c c p$. The quantity of $c c p$ transcript was not sufficient to generate a statistically significant signal above the background signal of the slide, and was filtered out at the pON filter stage of data analysis.

To confirm that the $c c p$ gene is regulated by FNR, and to compare the relative activity of the $c c p$ and aniA promoters, N. gonorrhoeae strains RUG7001, carrying a chromosomal aniA::lacZ fusion, and JCGC201 \& JCGC202, $\mathrm{fnr}^{+}$ and $f n r$ derivatives carrying $c c p:: l a c Z$, were grown microaerobically in gonococcal broth (GCB) in the presence and absence of nitrite and assayed for $\beta$-galactosidase activity after 4, 5, 6, and 7 hours of growth (Figure 2). Far less $\beta$-galactosidase activity accumulated in the ccp::lac $Z$ $f n r$ mutant strain than in the $f n r^{+}$strain, and the $\beta$-galactosidase activity of the $\mathrm{fnr}^{+}$strain was higher in the absence than in the presence of nitrite. During growth in the absence of nitrite, at an $\mathrm{OD}_{650}$ of around 0.5 (corresponding to $0.2 \mathrm{~g}$ bacterial dry weight $\mathrm{l}^{-1}$ ), FNR activated $\mathrm{P}_{c c p}$ 24-fold. These data support the previously reported observation that $c c p$ expression is repressed by the presence of nitrite in an $\mathrm{fnr}^{+}$strain [7]. Furthermore, expres- sion of $c c p$ was observed to be FNR-dependent in quantitative real time PCR experiments in which transcript levels in fnr mutant and wild-type strains were compared. Whereas in the parental strain $c c p$ expression was induced 18-fold during oxygen limited growth, there was no induction of $c c p$ expression in the fnr mutant strain. These data confirm that, while the ccp gene is not included in the list of FNR-activated genes from the microarray data due to low expression levels, the ccp promoter is activated by FNR. In addition, the ccp promoter was enriched in ChIP experiments, so was shown to bind FNR in vivo. However, these control $c c p$ experiments illustrate a rarely documented weakness of microarray experiments, namely the problem of false negative results that, due to overstringent use of statistical analysis, might lead to underestimation of the number of differentially expressed genes. It also illustrates the utility of an analytical approach that incorporates exclusion of data for transcripts that do not generate detectable signals; so that those genes below the detectable thresholds are readily identified, rather than using 'flooring' or other methods for filling in low intensity or empty microarray data fields.

\section{The gonococcal NarQP cannot complement an E. coli narQP mutation}

The microarray data revealed fundamental differences between the FNR regulons of E. coli and N. gonorrhoeae, not least in that the gonococcal FNR regulon is very small compared with its E. coli counterpart. However, in both organisms, expression of the major nitrite reductases is coactivated by FNR and a two-component regulatory system, NarQ-NarP, that bind to similar target sequences located at almost identical positions relative to the respective transcription start sites $\left(\mathrm{P}_{\text {aniA }}\right.$ in N. gonorrhoeae; $\mathrm{P}_{\text {nirB }}$ in E. coli $[5,6,14-18])$. We have previously demonstrated that a single amino acid substitution is sufficient to enable the gonococcal FNR to complement an E. coli fnr mutation. It was therefore of interest to determine whether the gonococcal NarQ-NarP could complement E. coli mutants defective in both NarXL and NarQP.

The gonococcal narQP genes were expressed in E. coli under the control of the E. coli fnr promoter from plasmid pGCNarQP, and the ability of the gonococcal NarQP proteins to activate the E. coli nirB promoter was assessed [19]. Expression of the E. coli nitrite reductase NirBD is activated by FNR in response to oxygen limitation and by NarQP in response to the availability of nitrate or nitrite [20]. E. coli strains JCB386 (nirB::lacZ), JCB3861 (nirB::lacZ narXL), JBC3863 (nirB::lacZ narXLQP), and JCB3863 transformed with pGCNarQP were grown anaerobically in the presence or absence of $5 \mathrm{mM} \mathrm{NaNO}_{2}$ or 20 $\mathrm{mM} \mathrm{NaNO} \mathrm{N}_{3}$ and $\beta$-galactosidase activities measured (Table 2A). 
A.

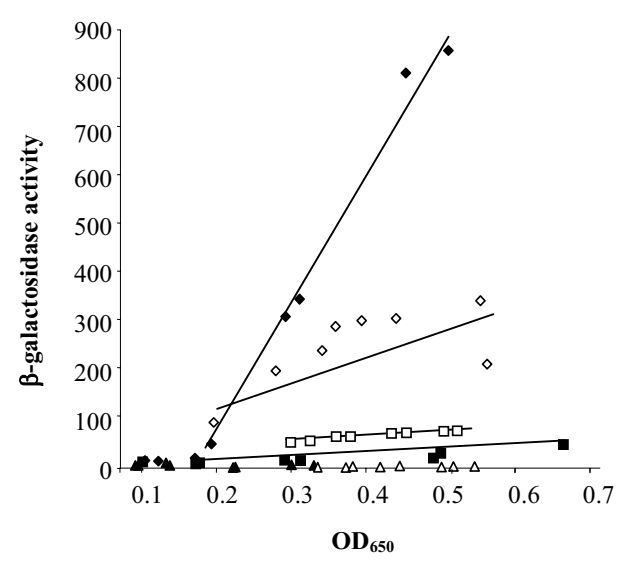

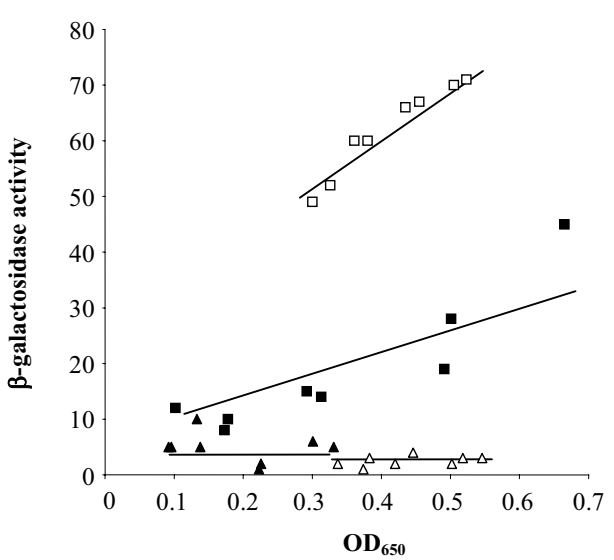

- aniA::lacZ+ $\mathrm{NO}_{2}^{-}$

- aniA::lacZ $-\mathrm{NO}_{2}^{-}$

- $c c p:: l a c Z+\mathrm{NO}_{2}^{-}$

口 cсp::lacZ $-\mathrm{NO}_{2}$

\ ccp::lacZfnr $+\mathrm{NO}_{2}$

$\triangle$ ccp : :lacZ fnr $-\mathrm{NO}_{2}^{-}$

\section{Figure 2}

A. $\beta$-galactosidase activity of the aniA::IacZ and ccp::IacZ fusions. Strains RUG700I (aniA::lacZ), JCGC20I (ccp::IacZ), and JCGC202 (ccp::lacZ fnr) were grown in liquid culture with or without $5 \mathrm{mM} \mathrm{NaNO}$. Samples were taken at hourly intervals, $\beta$ galactosidase activity measured, and the $\beta$-galactosidase activities plotted against optical density at $650 \mathrm{~nm}$. B. Data for the JCGC20I (ccp::lacZ) and JCGC202 (ccp::lacZ fnr) fusions are shown on an expanded scale to illustrate more clearly the effects of FNR and nitrite on ccp expression.

Table 2: Complementation of E. coli narXLQP mutations by gonococcal NarQ and NarQP.

\begin{tabular}{|c|c|c|c|c|c|c|}
\hline \multirow[t]{2}{*}{ Host strain } & \multirow[t]{2}{*}{ Host genotype } & \multirow[t]{2}{*}{ Plasmid } & \multirow[t]{2}{*}{ Reporter } & \multicolumn{3}{|c|}{$\beta$-galactosidase activity } \\
\hline & & & & $\varnothing_{2}$ & $+\mathrm{NO}_{2}^{-}$ & $+\mathrm{NO}_{3}$ \\
\hline \multicolumn{7}{|c|}{ A. Complementation of a narXLQP mutation } \\
\hline JCB386 & & none & nirB::lacZ & 120 & 650 & 1200 \\
\hline JCB386I & $n a r X L$ & none & nirB::IacZ & 80 & 130 & 570 \\
\hline JCB3863 & narXLQP & none & nirB::IacZ & 49 & 39 & 61 \\
\hline JCB3863 & narXLQP & PGCNarQP & nirB::IacZ & 64 & 67 & 81 \\
\hline \multicolumn{7}{|c|}{ B. Phosphorylation of E. coli NarP by gonococcal NarQ } \\
\hline JCB39I & $\operatorname{nar} X L Q$ & none & napF::lacZ & 130 & 110 & 120 \\
\hline JСB391 & narXLQ & pBADgcQ & napF::lacZ & 1630 & 1390 & 1350 \\
\hline \multicolumn{7}{|c|}{ C. Phosphorylation of E. coli NarL by gonococcal NarQ } \\
\hline $\mathrm{JCBI} 2$ & narXQ & none & frdA::IacZ & 2830 & 2070 & 2570 \\
\hline $\mathrm{JCBI} 2$ & narXQ & pBADgcQ & frdA::IacZ & 780 & 650 & 820 \\
\hline
\end{tabular}

E. coli transformed with the stated plasmids was grown anaerobically in LB in A. and B. and in minimal medium in C. in the absence or presence of $2.5 \mathrm{mM} \mathrm{NaNO}_{2}$ or $20 \mathrm{mM} \mathrm{NaNO}_{3}$ and the $\beta$-galactosidase activities determined. 
In E. coli strain JCB386, the nirB promoter is activated in response to both nitrite and nitrate. Activation in the presence of nitrite is reduced in strain JCB3861, which lacks NarXL. In the narXLQP strain JCB3863, nirB activity is very low in all three growth conditions, since NarQP is required for activation in the presence of nitrite or nitrate. When transformed with pGCNarQP, strain JCB3863 has similarly low $\beta$-galactosidase activities in all three conditions. Expression of the gonococcal narQP genes from pGCNarQP was verified by RT-PCR (data not shown). Therefore, it was concluded that gonococcal NarQP cannot complement an E. coli narXLQP mutation at $\mathrm{P}_{\text {nirB }}$.

\section{Ligand sensing and signal transduction characteristics of the gonococcal NarQ}

Only a very limited range of genetic techniques are available to investigate sensor kinases and response regulators by site-directed mutagenesis and gene deletions in the gonococcus. However, as some sensor kinases have been shown to phosphorylate response regulators of a heterologous host $[21,22]$, the ability of gonococcal NarQ to phosphorylate the E. coli NarP protein was assessed to investigate the ligand sensing and signal transduction characteristics of the gonococcal NarQ and NarP proteins. Strain JCB391 (narXL narQ) and JCB391 transformed with pBADgcQ, expressing gonococcal narQ, each cotransformed with pRNW15 carrying napF::lacZ, were grown anaerobically in the presence or absence of nitrate and nitrite and their $\beta$-galactosidase activities determined (Table 2B). The NarP-dependent napF promoter was not activated during growth in the presence of nitrite and nitrate in strain JCB391, since the NarQ sensor kinase was not present and NarP was unable to become phosphorylated, but was activated constitutively in strain JCB391 expressing the gonococcal NarQ from pBADgcQ. The explanation for this observation is that the gonococcal NarQ was constitutively phosphorylating the E. coli NarP protein, which was activating transcription. This was the first indication that the gonococcal NarQ sensor kinase might be ligand-insensitive and constitutively active in $E$. coli.

Expression of the E. coli fumarate reductase operon, frdA$B C D$, is activated by FNR in response to oxygen limitation but repressed by NarL in response to the availability of nitrate or nitrite [23]. Due to the absence of a 7-2-7 inverted repeat sequence (where the 7 bases are the NarL heptamer), NarP is unable to bind at this promoter [24]. If the gonococcal NarQ is constitutively active, it should also be able to activate E. coli NarL and hence repress transcription at $\mathrm{P}_{\text {frd }}$. Strain JCB12 (frdA::lacZ narXQ) and strain JCB12 transformed with pBADgcQ expressing gonococcal NarQ, were grown anaerobically in the presence or absence of nitrate and nitrite and their $\beta$-galactosidase activities were determined (Table 2C). The frdA promoter was activated in all three conditions in strain JCB12, but was repressed in all three conditions by strain JCB12 expressing the gonococcal NarQ from $\mathrm{pBADgcQ}$, as expected if the gonococcal NarQ was continually phosphorylating the E. coli NarL protein and therefore constitutively active in E. coli.

\section{Mutations in the P-box of E. coli NarQ do not alter ligand specificity}

Previous studies have revealed residues in a periplasmic region of the E. coli NarQ and NarX proteins, the P-box, that are important for ligand sensing and discrimination between nitrate and nitrite [25-28]. Only ten of the 18 residues that comprise the gonococcal P-box are the same as those of either the E. coli NarQ or NarX proteins. Four residues are proposed to be important for ligand discrimination in E. coli NarX (H45, K49, R54 and R59); substitutions at any of these residues result in NarX proteins with altered ligand sensing characteristics [28]. Of these four, only R59 is conserved in the gonococcal NarQ. The residue in gonococcal NarQ corresponding to R54 is a lysine; an $\mathrm{R} 54 \mathrm{~K}$ substitution in $E$. coli NarX results in a ligand-insensitive phenotype. To determine whether the differences in the P-box of gonococcal NarQ determined nitrite specificity, the E. coli NarQ P-box was mutated to resemble the gonococcal P-box (Table 3). Mutated NarQ proteins were expressed in E. coli strains mutated in narX and narQ and their ability to activate nirB::lacZ or repress frdA::lac $Z$ chromosomal fusions was assessed using $\beta$ galactosidase assays (Table 3). Substitutions in both single and multiple residues resulted in NarQ proteins that were inactive and ligand insensitive, a "locked-off" phenotype.

\section{Discussion \\ Contrasts between the $\mathbf{N}$. gonorrhoeae and E. coli FNR and NarP regulons}

The first conclusion from this study is that the N. gonorrhoeae FNR and NarP regulons are both much smaller than their E. coli counterparts. Constantinidou et al. [8] estimated that at least 104 , and possibly a many as $115, E$. coli operons are regulated directly by FNR, including 68 that are induced, and 36 that are repressed: the FNR regulon of the pathogenic E. coli O157 is of a similar size (Overton, Constantinidou and Cole, unpublished data). The corresponding figures for the gonococcus are that at most 14 transcripts are induced by FNR, and 6 are repressed. Based upon DNA sequence analysis and ChIP experiments, even this might be an over-estimate of the genes that are directly regulated by FNR. However, it is similar to the 9 transcription units recently proposed to be activated by FNR in the closely related pathogen, Neisseria meningitidis [29]. These authors derived a consensus sequence for meningococcal FNR-binding sites that differed at one position from the consensus FNR-binding site 
Table 3: P-box substitutions in E. coli NarQ.

\begin{tabular}{lccc}
\hline Plasmid & Substitutions & P-box sequence & Phenotype \\
\hline PBADEcQ & E. coli NarQ P-box & DAEAINIAGSLRMQSYRL & Nitrate and nitrite sensing \\
PRNW18 & R54K & DAEAINIAGSLKMQSYRL & Ligand insensitive \\
PRNW19 & N48E I49E & DAEAIEEAGSLRMQSYRL & Ligand insensitive \\
PRNW20 & N48E I49E R54K & DAEAIEEAGSLKMQSYRL & Ligand insensitive \\
PRNW29 & D43A E45S A46V N48E I49E & AASVIEEAGSLRMQSYRL & Ligand insensitive \\
PRNW30 & D43A E45S A46V & AASVINIAGSLRMQSYRL & Ligand insensitive \\
PRNW31 & D43A E45S A46V R54K & AASVINIAGSLKMQSYRL & Ligand insensitive \\
PRNW32 & D43A E45S A46V N48E I49E R54K & AASVIEEAGSLKMQSYRL & Ligand insensitive \\
PRNW33 & D43A E45S A46V S52N S57A & AASVINIAGNLRMQAYRL & Ligand insensitive \\
PRNW34 & D43A E45S A46V N48E I49E S52N R54K S57A & AASVIEEAGNLKMQAYRL &
\end{tabular}

E. coli strains JCB39I (narXL narQ) or JCBI2 (narXL narQ frdA::IacZ) transformed with pBADecQ expressing wild-type or mutated NarQ proteins were grown anaerobically in $\mathrm{LB}$ in the absence or presence of $20 \mathrm{mM} \mathrm{NaNO}$ and the $\beta$-galactosidase activities determined. Mutations in the $\mathrm{P}$ box are shown in bold type. Wild-type NarQ was able to phosphorylate NarP in the presence of nitrate, activating expression of nirB and repressing frdA. Mutated NarQ proteins were inactive.

in E. coli. However, three lines of evidence indicate that the consensus gonococcal FNR binding site is identical to that of E. coli FNR: (i) there is a perfect match to the E. coli consensus sequence in the regulatory region of the N. gonorrhoeae transcript that is most dependent upon FNR; (ii) Overton et al. [14] showed that a single amino acid substitution, S18F in the N-terminal domain, which is located well away from the DNA recognition helix, enables the gonococcal FNR to activate a range of FNR-dependent promoters as effectively as the E. coli FNR, suggesting that they have similar site specificities; and (iii) unsubstituted gonococcal FNR can function as a repressor at $E$. coli FNRbinding sites, again suggesting that they have similar, or even identical, specificities [14].

The $c c p$ promoter was not identified in the microarray study to be part of the FNR regulon, raising the question whether other members of the gonococcal FNR regulon had been missed because they also are expressed at a level below the threshold set in this analysis. However, close inspection of the raw data failed to reveal additional candidates that, like $c c p$, were false negatives. Nevertheless, the ccp example provides clear evidence that false negative results, like false positive results, can be a problem in microarray analysis.

We recently showed that the list of transcripts differentially expressed in a gonococcal narP mutant and its parent is even smaller than the corresponding list for the FNR regulon, and inverted repeat sequences similar to the binding site for $E$. coli NarP were readily identified in only four promoter regions [10]. The N. gonorrhoeae NarP is only distantly related to the E. coli NarP or NarL (42\% and $41.5 \%$ sequence identity, respectively), so although gonococcal NarP can recognise and bind to the same inverted repeat sequence as $E$. coli $\mathrm{NarP}$, it is not surprising that it cannot functionally complement E. coli narXL or narQP mutants. Apart from genes involved in denitrification, only two transcripts encoding proteins of unknown function were regulated by NarP, unlike in E. coli in which there is an extensive regulon of genes involved in anaerobic metabolism that are repressed by nitrate-activated NarP [8].

The proposal that NarQ from N. gonorrhoeae is a ligand insensitive sensor kinase locked on in its kinase mode is also fully supported by experiments presented in Tables 2 and 3, consistent with the fundamental differences in nitrate and nitrite sensing between the two types of bacteria. E. coli NarQ is exquisitely sensitive to nitrate, but two orders of magnitude less sensitive to nitrite [30]. In contrast, gonococcal NarQ is insensitive to both nitrate and nitrite, and induction of aniA transcription in the presence of nitrite requires inactivation of the repressor, NsrR, not by nitrite but by the product of nitrite reduction, nitric oxide [10]. As gonococci lack the alternative electron transfer pathways that in E. coli are subject to NarP repression and that they are unable to metabolise nitrate, it is entirely consistent that they also lack a nitrate-sensing two-component regulatory system. Consequently, data in Table 2 show that gonococcal NarQ can constitutively phosphorylate E. coli NarL or NarP. Conversely, amino acid substitutions that make the P box of E. coli NarQ more like that of the gonococcal NarQ simply inactivate signal transduction (Table 3 ), as had been found in previous detailed site-directed mutagenesis experiments of $E$. coli NarX [25-28].

\section{Conclusion}

Both E. coli and N. gonorrhoeae are Gram-negative human pathogens that show adaptation to, and are able to live in, anaerobic niches as part of their normal colonization- 
transmission cycles within the host. Both species utilize a common regulator, FNR, in the control of this response. However, this study shows that whereas a wide range of responses and physiological adaptations are coordinated by this FNR in E. coli, the adaptations are far fewer and are specifically and primarily focussed upon the immediate metabolic needs for utilising alternate electron acceptors under anaerobic conditions in N. gonorrhoeae. As such, while this regulator controls what appears to be a fully integrated response in $E$. coli, in $N$. gonorrhoeae the response is essentially independent of the other physiological changes required for adaptation to anaerobic growth. Furthermore, differences between the ligand sensing and signal transduction capabilities of the E. coli and N. gonorrhoeae NarQ-NarP proteins were revealed. This illustrates fundamental differences between the way in which environmental responses are controlled and integrated in these two species, and highlights the importance of specific investigations of species with different adaptation strategies.

\section{Methods}

Strains, plasmids, oligonucleotide primers, and gene identification numbers used in this study

The strains and plasmids used in this study are listed in Table 4. Sequences of oligonucleotide primers are available see Additional file 1. The XNG gene identification numbers used for some genes in this study (those not annotated in the GenBank database) refer to the genome sequence annotation of $N$. gonorrhoeae strain FA1090 that was used in the design of the pan-Neisseria microarray [31] and pan-Neisseria microarray-v2 [32]. A GBrowse database containing this annotation comparatively presented against other neisserial genome annotations can be found at http://www.compbio.ox.ac.uk/data. COG identifications were made using the NCBI Conserved Domain Search tool [33].

\section{Growth of $\mathbf{N}$. gonorrhoeae}

N. gonorrhoeae was grown on gonococcal agar plates and in gonococcal broth (GCB) supplied by BD. Solid and liquid media were supplemented with $1 \%(\mathrm{v} / \mathrm{v})$ Kellogg's Supplement [34]. For liquid cultures, $2 \mu \mathrm{l}$ of a stock of $N$. gonorrhoeae was plated onto a gonococcal agar plate and incubated in a candle jar at $37^{\circ} \mathrm{C}$ for 24 hours. Bacteria from this plate were swabbed onto a second plate and incubated in the same way for a further 16 hours. The entire bacterial growth from this second plate was swabbed into $10 \mathrm{ml}$ of GCB and incubated at $37^{\circ} \mathrm{C}$ in an orbital shaker at $100 \mathrm{rpm}$ for one hour. This $10 \mathrm{ml}$ preculture was then tipped into $50 \mathrm{ml}$ of GCB in a $100 \mathrm{ml}$ conical flask and incubated in the same way. For growth with nitrite, the pre-culture was supplemented with 0.5 $\mathrm{mM} \mathrm{NaNO}_{2}$ and the flasks were supplemented with $5 \mathrm{mM}$ $\mathrm{NaNO}_{2}$.

\section{Preparation of RNA for microarray experiments}

Samples $(10 \mathrm{ml})$ of bacterial culture were mixed with an equal volume of RNAlater (Ambion), the bacteria were pelleted by centrifugation, resuspended in $0.5 \mathrm{ml}$ of RNAlater and stored at $4^{\circ} \mathrm{C}$ overnight. Bacteria were collected by centrifugation and resuspended in TRIzol (Invitrogen) by vortexing for ten minutes. Chloroform was added, the phases were separated and the aqueous phase was transferred to a clean tube. Crude RNA in the aqueous phase was precipitated with isopropanol and cleaned using an RNeasy kit (QIAGEN). Purified RNA was eluted in RNase-free water with $2 \%(\mathrm{v} / \mathrm{v})$ SuperaseIN RNase inhibitor (Ambion).

\section{cDNA generation, labelling, and microarray hybridisation} Reagents and enzymes for the preparation of materials for microarray hybridisations were sourced from the 3DNA Array 900 MPX kit (Genisphere, PA, USA) unless otherwise stated. One microgram of RNA was reverse transcribed into unlabelled cDNA using SuperScript III reverse transcriptase (Invitrogen) at $42^{\circ} \mathrm{C}$ for two hours. The cDNA was cleaned using a Clean \& Concentrate- 5 column (Zymo Research) and poly-T tailed with terminal deoxynucleotidyl transferase. Dye-specific capture sequences were ligated to the poly-T tails and the tagged cDNAs were cleaned using a Clean \& Concentrate-5 column. The panNeisseria microarray v-2 [32], containing probes to N. gonorrhoeae and $N$. meningitidis genes, was used for these experiments. Microarray slides were pre-hybridised in 3.5 $\times$ SSC, $0.1 \%$ SDS and $10 \mathrm{mg} \mathrm{mL}^{-1} \mathrm{BSA}$ for $65^{\circ} \mathrm{C}$ for 20 minutes, washed with water and isopropanol, dried with an airbrush, and pre-scanned to check for array defects. The capture sequence tagged cDNAs were hybridised onto the microarray slide for $16 \mathrm{~h}$ at $60^{\circ} \mathrm{C}$ in a SlideBooster with the power setting at 25 and a pulse/pause ratio of 3:7. Following the first hybridisation, the slides were washed in $2 \times \mathrm{SSC}, 0.2 \% \mathrm{SDS}$ for $10 \mathrm{~min}$. at $60^{\circ} \mathrm{C}$, followed by washes at $2 \times$ SSC and $0.2 \times$ SSC for ten minutes, each at room temperature. The slides were dried with an airbrush and hybridised with the Cy 3 and Cy 5 capture reagents at $55^{\circ} \mathrm{C}$ for $4 \mathrm{~h}$ in a SlideBooster. The slides were again washed in $2 \times \mathrm{SSC}, 0.2 \% \operatorname{SDS}\left(10 \mathrm{~min}\right.$. at $60^{\circ} \mathrm{C}$ ) followed by $10 \mathrm{~min}$. room temperature washes in $2 \times$ SSC and 0.2 $\times$ SSC (10 min. at room temperature) and dried with an airbrush. Dried slides were scanned using a ScanArray ExpressHT (Perkin Elmer) using autocalibration. For slides PNA6_29 - PNA6_39 this scanner was unavailable and the image data was collected using a GenePix 4000B (Axon Instruments) and manual calibration.

\section{Microarray data analysis}

Where necessary, scanned microarray images were straightened with ImageViewer (BlueGnome). Images were analysed using BlueFuse for Microarrays (BlueGnome). Spot data were extracted from images and manu- 
Table 4: Strains and plasmids used in this study.

\begin{tabular}{|c|c|c|}
\hline Strain & Description & Source \\
\hline \multicolumn{3}{|c|}{ N. gonorrhoeae strains } \\
\hline F62 & Parental strain & Laboratory stocks \\
\hline RUG700I & F62 proAB paniA::IacZ & {$[43]$} \\
\hline RUG7022 & F62 proAB paniA::lacZ fnr & [6] \\
\hline JCGC20I & F62 proAB pccp::lacZ & [5] \\
\hline JCGC202 & F62 proAB pсcp:::lacZ fnr & [5] \\
\hline JCGC502 & F62 fnr-3xFLAG Kan ${ }^{R}$ & This work \\
\hline \multicolumn{3}{|l|}{ E. coli strains } \\
\hline RV & Parental strain & Laboratory stocks \\
\hline JCB386 & $\Delta$ (nirB-cycG) pnirB::IacZ & [44] \\
\hline JCB386I & JCB386 narXL & This work \\
\hline JCB3862 & JCB386 narXL narQ & This work \\
\hline JCB3863 & JCB386 narXL narQ narP & This work \\
\hline JCB39I & $\mathrm{RV}$ narXL narQ pcnB & This work \\
\hline $\mathrm{JCBI} 2$ & RV narX narQ frdA::lacZ & {$[10]$} \\
\hline \multicolumn{3}{|l|}{ Plasmids } \\
\hline pGCFNR3 & Gonococcal fnr, under the regulation of the $E$. coli fnr promoter, cloned into pBR322. & [5] \\
\hline PGCFNRi & PGCFNR3 with $\mathrm{Kpnl}$ and $\mathrm{Xhol}$ restriction sites engineered downstream of the fnr gene & This work \\
\hline PGCFNR-FLAG & PGCFNR3 with a 3xFLAG tag and Kanamycin resistance cassette inserted downstream of the fnr gene & This work \\
\hline PSUBII & Epitope tagging plasmid carrying 3xFLAG tag and kanamycin resistance cassette. & [36] \\
\hline PGCNarQP & Gonococcal narQP genes under the control of the E. coli fnr promoter cloned into pBR322. & This work \\
\hline pBADgcQ & Gonococcal narQ gene under the control of the araBAD promoter cloned into pBAD myc-His $\mathrm{A}$. & This work \\
\hline pBADecQ & E. coli narQ gene under the control of the araBAD promoter cloned into pBAD myc-His $A$ & This work \\
\hline pRNWI8 & PBADEcQ containing R54K substitution in narQ & This work \\
\hline PRNWI9 & PBADEcQ containing N48E I49E substitution in narQ & This work \\
\hline pRNW20 & pBADEcQ containing N48E I49E R54K substitution in narQ & This work \\
\hline PRNW29 & PBADEcQ containing D43A E45S A46V N48E I49E substitution in narQ & This work \\
\hline pRNW30 & pBADEcQ containing D43A E45S A46V substitution in narQ & This work \\
\hline PRNW3I & pBADEcQ containing D43A E45S A46V R54K substitution in narQ & This work \\
\hline pRNW32 & PBADEcQ containing D43A E45S A46V N48E I49E R54K substitution in narQ & This work \\
\hline PRNW33 & PBADEcQ containing D43A E45S A46V S52N S57A substitution in narQ & This work \\
\hline pRNW34 & pBADEcQ containing D43A E45S A46V N48E I49E S52N R54K S57A substitution in narQ & This work \\
\hline p7I50 & E. coli nirB promoter cloned into the lacZ reporter vector pRW50. & [19] \\
\hline PRNWI5 & E. coli napF promoter cloned into the lacZ reporter vector pRW50. & This work \\
\hline
\end{tabular}

ally flagged to remove artefacts before fusion. Fused data were filtered according to pON value [35]. Spots with pON values less than 0.5 in both channels were excluded to eliminate the bias generated by the inclusion of unhybridized spots in the statistical interpretation of the data, and the data globally adjusted such that the mean rRNA ratio was 1.0. The data were then analysed using BASE. For each pair wise comparison, gene expression median foldchanges were calculated from the biological replicates using the MGH fold-change algorithm, and the Student's t-test was used to assess statistical significance. Since six biological replicates were analysed, a $p$ value of 0.01 was used. Genes whose transcript levels did not change consistently (i.e. more or less abundant in the mutant compared to the parental strain) in all the biological replicates in which they were detected for each experiment were discarded. Data were also analysed using a locally prepared implementation of the Cyber-T algorithm within BASE; the results from this analysis is available online at http:// tinyurl.com/fu2um. Total microarray data have been deposited in the ArrayExpress database http:// www.ebi.ac.uk/arrayexpress/ with the accession number E-MEXP-726.

\section{Generation of a chromosomal FNR-3xFLAG fusion in N. gonorrhoeae}

Codons for a 3x FLAG tag, (DYKDDDDK)3, were linked in-frame to the 3' end of the fnr gene on the chromosome of $N$. gonorrhoeae strain F62. Plasmid pGCFNR3 contains the gonococcal $f n r$ gene and 500 bp of downstream sequence under the control of the E. coli fnr promoter [5]. Inverse PCR, using primers FNRiPCRFwd and FNRiPCRRwd was used to introduce a KpnI restriction site in place of the $f n r$ gene stop codon and an Xhol site immediately downstream of the $f n r$ gene, yielding plasmid pGCFNRi. Sequences of all oligonucleotide primers used in this study are available on-line in Table S1. The kanamycin resistance cassette and the 3x FLAG tag encoded by pSUB11 [36] were amplified by PCR using primers FLAGFwd and FLAGRwd, which introduced KpnI and XhoI 
sites at either end of the resultant fragment. The $\operatorname{Kan}^{\mathrm{R}}-3 \mathrm{x}$ FLAG fragment and pGCFNRi were digested with KpnI and XhoI, the plasmid fragment was dephosphorylated with calf alkaline phosphatase, and the two fragments were ligated to form plasmid pGCFNR-FLAG. Western blotting was used to show that pGCFNR-FLAG expressed a $30 \mathrm{kDa}$ FLAG-tagged protein in E. coli, corresponding to the gonococcal FNR. To transfer the $f n r-3 x$ FLAG-Kan ${ }^{R}$ fragment into $N$. gonorrhoeae, pGCFNR-FLAG was digested with HindIII and Bam HI and the $3.5 \mathrm{~kb}$ fnr-3x FLAG-Kan ${ }^{\mathrm{R}}$ fragment was purified by phenol chloroform extraction and ethanol precipitation. Piliated N. gonorrhoeae strain F62 was transformed with this linear DNA fragment, which recombined with the fnr locus on the gonococcal chromosome yielding strain JCGC502. To confirm that the FLAG-tagged FNR protein was still functional and able to activate aniA expression, the ability of strain JCGC502 to utilise nitrite was determined. Cultures of JCGC502 were grown microaerobically in the presence and absence of nitrite and optical densities were measured at hourly intervals. Strain JCGC502 grew exponentially and respired nitrite, therefore AniA was expressed and the FLAG-tagged FNR protein was functional. In addition, samples taken from the cultures at hourly intervals were probed by Western blotting to determine the quantity of FNR-3xFLAG present (see Additional file 2). No significant differences were observed either over the course of the growth curves or between cultures grown in the presence or absence of nitrite. These data confirm that gonococcal FNR activity is likely to be modulated by oxygen in a manner similar to the E. coli FNR protein, rather than expression level, as it the case of some other CRP-FNR superfamily members such as Bradyrhizobium japonicum FixK $_{2}$ [37].

\section{Western blotting}

Gonococcal proteins separated by Tris/Tricine SDS-PAGE using a 15\% polyacrylamide gel were blotted onto a PVDF membrane and FLAG-tagged FNR protein was detected using anti-FLAG monoclonal antibodies (Sigma) and the ECL-Plus chemiluminescence detection system (GE Healthcare Life Sciences).

\section{Chromatin Immunoprecipitation}

Interactions between FNR and promoter DNA were studied in vivo by Chromatin Immunoprecipitation (ChIP) as described by Grainger et al. [38]. N. gonorrhoeae strain JCGC502 was grown microaerobically with or without 5 $\mathrm{mM} \mathrm{NaNO}$ to late exponential phase. Protein-DNA crosslinking, chromatin preparation, and immunoprecipitations were as described previously except that the tagged protein was immunoprecipitated with anti-FLAG monoclonal antibodies (Sigma) for $16 \mathrm{~h}$ at $4{ }^{\circ} \mathrm{C}$. The concentration of immunoprecipitated promoter fragments was measured using quantitative real time PCR [39].
Primers for each promoter were designed using PrimerExpress (Applied Biosystems) and are listed in Table S1. The promoter of the hpt (NG2035) gene, which is not regulated by FNR and is not preceded by an FNR binding site, was a negative control used to normalise the data. Promoter fragments enriched by $60 \%$ or more in at least two independent ChIP experiments, relative to the $h p t$ promoter fragment, were scored positive.

\section{Construction of E. coli strains}

The narXL genes were deleted from E. coli strain JCB386 using the gene-replacement method [36]. Primers EcnarXp1 and EcnarLp2 were used to amplify the chloramphenicol acetyltransferase gene from plasmid pKD3 [40] resulting in a cat cassette flanked by DNA with sequence homology to upstream and downstream of the narXL genes. This linear DNA fragment was electroporated into strain E. coli JCB386 containing pKD46, encoding the $\lambda$ Red recombinase, which mediated recombination of the chloramphenicol resistance cassette into the E. coli chromosome and replacement of the narXL genes with the cat gene. Transformation of the narXL::chl ${ }^{\mathrm{R}}$ strain with pCP20, encoding FLP recombinase, resulted in loss of the chloramphenicol resistance gene and creation of an unmarked narXL deletion in strain JCB3861. The narQ deletion was generated by the same method, using primers EcnarQp1 and EcnarQp2, resulting in strain JCB3862 (narXL narQ). The narP deletion was transferred using P1 transduction; strain JCB3862 was transduced with bacteriophage P1 that had been propagated on E. coli strain JCB3875 which carries a narP::chl ${ }^{\mathrm{R}}$ mutation [17], generating strain JCB3863 (narXL narQ narP). Strain JCB391 was generated by successive transduction of the narXL::chl ${ }^{\mathrm{R}}$ and $n a r Q:: \mathrm{chl}^{\mathrm{R}}$ mutations into strain RV followed by removal of the antibiotic resistance cassettes using pCP20. The pcnB:::kan ${ }^{\mathrm{R}}$ mutation, effectively reducing the plasmid copy number to one, was transferred from strain RP7974 [41].

\section{Construction of plasmids expressing gonococcal and $\mathrm{E}$. coli narQP}

The gonococcal narQP genes were amplified from chromosomal DNA by PCR using primers NgNarQPNcoI and NgNarQPBamHI, generating a fragment with $\mathrm{NcoI}$ and BamHI sites at each end. The resultant PCR product and pGCFNR3 were both digested with $\mathrm{NcoI}$ and BamHI and ligated, yielding $\mathrm{pGCNarQP}$. For plasmid $\mathrm{pBADgcQ}$, the gonococcal narQ gene was cloned into the arabinoseinducible $\mathrm{pBAD} m y c$-hisA overexpression vector using primers NgNarQNcoI and NgNarQHindIII to generate an $\mathrm{NcoI}$ - HindIII narQ fragment, which was ligated into NcoI -HindIIIdigested pBAD $m y c$-hisA (Invitrogen). Similarly, pBADecQ contained the E. coli narQ gene cloned into pBAD $m y c$-hisA. Primers EcNarQ NcoI and EcNarQ BamHI 
were used to generate a NcoI-BamHI E. coli narQ fragment, which was cloned into pBAD $m y c$-hisA.

The Quikchange site-directed mutagenesis system (Stratagene) was used to generate specific mutations in the P-box region of the $E$. coli narQ gene using primers listed in Table S1: Primer pair SDM1 R-K FWD \& RVS were used to generate substitution R54K; pair SDM2 NI-EE FWD \& RVS substitutions N48E \& I49E; SDM3 DAEA-AASV FWD \& REV substitutions D43A E45S \& A46V ; SDM4 DAEAAASV FWD \& RVS substitutions D43A E45S \& A46V; and SDM5 SS-NA FWD \& RVS substitutions S52N \& S57A. Substitutions were combined by stepwise mutagenesis in plasmids pRNW18-34 as listed in Table 4.

\section{$\beta$-galactosidase assay}

E. coli was grown at $37^{\circ} \mathrm{C}$ or $30^{\circ} \mathrm{C}$ in LB (Luria-Bertani) medium with $0.4 \%$ glucose or in minimal medium [42] supplemented with $40 \mathrm{mM}$ sodium fumarate, $10 \% \mathrm{LB}$ and $0.4 \%$ glycerol. Where stated, cultures were supplemented with $20 \mathrm{mM} \mathrm{NaNO}_{3}$ or $2.5 \mathrm{mM} \mathrm{NaNO}_{2}$. Two ml aliquots of bacterial cultures were lysed by the addition of $30 \mu \mathrm{l}$ each toluene and $2 \%(\mathrm{w} / \mathrm{v})$ sodium deoxycholate and aerated at $30^{\circ} \mathrm{C}$ for 20 minutes. Lysates were assayed for $\beta$-galactosidase activity as previously described [15].

\section{Sequence pattern searching}

Potential FNR binding sites were located in promoter regions using Findpatterns in the GCG suite (Accelrys, Cambridge, UK) using the consensus E. coli FNR binding site, TTGATNNNNATCAA, to search the gonococcal DNA sequences.

\section{Abbreviations}

ChIP, Chromatin Immunoprecipitation; FNR, regulator of fumarate and nitrate reduction; NsrR, nitrosative stress response regulator; qRT-PCR, quantitative reverse transcriptase polymerase chain reaction; HAMP, histidine kinase, adenylate cyclase, methyl-accepting protein and phosphotransferase domain; TMII, second transmembrane region; RNS, Reactive Nitrogen Species; ROS, Reactive Oxygen Species; ; OMP, outer membrane protein; CDS, coding sequence.

\section{Authors' contributions}

RW, TWO and JAC designed the experimental work. RW and TWO performed the experimental work and analysed the data. SM developed and implemented the use of GBrowse for the display of the microarray data. LASS \& NJS designed and manufactured the PNAv2 microarrays and supervised the microarray-based experiments and analysis. RW, TWO, LASS, HS, JAC and NJS drafted the manuscript. All authors read and approved the final manuscript.

\section{Additional material}

\section{Additional file 1}

Primers used in this study. Primer sequences used for construction of plasmids and strains, mutagenesis, and quantitative real-time PCR Click here for file

[http://www.biomedcentral.com/content/supplementary/1471-

2164-8-35-S1.doc]

\section{Additional file 2}

Figure of growth curves of JCGC501 and anti FNR-3xFLAG Western blots. Figure showing: A. Growth characteristics of strain JCGC501, carrying a chromosomal fnr-3xFLAG fusion. Strain JCGC501 was grown microaerobically in the presence or absence of $5 \mathrm{mM} \mathrm{NaNO}_{2}$. B. Western blotting shows that the quantity of FNR-3xFLAG protein remains constant through the growth cycle. Samples were taken from the above growth curve at hourly intervals, separated by SDS-PAGE, blotted onto PVDF membrane and FNR-3xFLAG protein was detected using anti-FLAG antibodies and ECF-Plus chemiluminescent labelling.

Click here for file

[http://www.biomedcentral.com/content/supplementary/14712164-8-35-S2.pdf]

\section{Acknowledgements}

The authors thank D. Grainger and S. Busby for help with the ChIP experiments and for helpful discussions, and A. Jones for use of the Birmingham School of Biosciences genomics and proteomics facilities. This study was funded by the UK Biotechnology and Biological Sciences Research Council (BBSRC) Project Grant P21080, and by an MRC PhD training studentship to RNW. The microarray printing and other facilities were supported by funding from the EPA Cephalosporin Trust. The microarray data analysis and LIMS facilities and support were provided by the Dunn School/Weatherall Institute of Molecular Medicine Computational Biology Research Group.

\section{References}

I. Burnakis TG, Hildebrandt NB: Pelvic inflammatory disease: a review with emphasis on antimicrobial therapy. Rev Infect Dis 1986, 8:86-116.

2. Clark VL, Knapp JS, Thompson S, Klimpel KW: Presence of antibodies to the major anaerobically induced gonococcal outer membrane protein in sera from patients with gonococcal infections. Microb Pathog 1988, 5:38I-90.

3. Knapp JS, Clark VL: Anaerobic growth of Neisseria gonorrhoeae coupled to nitrite reduction. Infect Immun 1984, 46: I76-I8I.

4. Clark VL, Campbell LA, Palermo DA, Evans TM, Klimpel KW: Induction and repression of outer membrane proteins by anaerobic growth of Neisseria gonorrhoeae. Infect Immun 1987, 55:1359-64.

5. Lissenden S, Mohan S, Overton T, Regen T, Crooke H, Cardinale JA, Householder TC, Adams P, O'Connor CD, Clark VL, Smith H, Cole JA: Identification of transcription activators that regulate gonococcal adaptation from aerobic to anaerobic or oxygenlimited growth. Mol Microbiol 2000, 37:839-855.

6. Householder TC, Belli WA, Lissenden S, Cole JA, Clark VL: cis- and trans-Acting Elements Involved in Regulation of aniA, the Gene Encoding the Major Anaerobically Induced Outer Membrane Protein in Neisseria gonorrhoeae. J Bacteriol 1999, 18I:54I-55I.

7. Turner S, Reid E, Smith H, Cole JA: A novel cytochrome c peroxidase from Neisseria gonorrhoeae: a lipoprotein from a Gramnegative bacterium. Biochem J 2003, 373:865-873.

8. Constantinidou CC, Hobman JL, Patel MD, Penn CW, Cole JA, Overton TW: A reassessment of the fumarate and nitrate reduc- 
tion regulon and transcriptomic analysis of the effects of nitrate, nitrite, NarXL and NarQP as Escherichia coli adapts from aerobic to anaerobic growth. J Biol Chem 2006, 28 I:4802-4808.

9. Mey AR, Craig SA, Payne SM: Characterization of Vibrio cholerae RyhB: the RyhB regulon and role of RyhB in biofilm formation. Infect Immun 2005, 73:5706-5709.

10. Overton TW, Whitehead R, Li Y, Snyder LA, Saunders NJ, Smith H, Cole JA: Coordinated regulation of the Neisseria gonorrhoeae truncated denitrification pathway by the nitric oxide-sensitive repressor, NsrR, and nitrite-insensitive NarQ-NarP. J Biol Chem 2006, 281:331।15-33126.

II. Raskin DM, de Boer PA: MinDE-dependent pole-to-pole oscillation of division inhibitor MinC in Escherichia coli. J Bacteriol 1999, 181:6419-6424.

12. Householder TC, Fozo EM, Cardinale JA, Clark VL: Gonococcal Nitric Oxide Reductase Is Encoded by a Single Gene, norB, Which Is Required for Anaerobic Growth and Is Induced by Nitric Oxide. Infect Immun 2000, 68:524I-5246.

13. Stohl EA, Criss AK, Seifert HS: The transcriptome response of Neisseria gonorrhoeae to hydrogen peroxide reveals genes with previously uncharacterized roles in oxidative damage protection. Mol Microbiol 2005, 58:520-32.

14. Overton T, Reid EGF, Foxall R, Smith H, Busby SJW, Cole JA: Transcription activation at Escherichia coli FNR-dependent promoters by the gonococcal FNR protein: Effects of a novel SI8F substitution and comparisons with the corresponding substitution in E. coli FNR. J Bacteriol 2003, 185:4734-4747.

15. Jayaraman P-S, Peakman T, Busby SJW, Quincey R, Cole JA: Location and sequence of the promoter of the gene for the NADHdependent nitrite reductase of Escherichia coli and its regulation by oxygen, the FNR protein and nitrite. J Mol Biol 1987, 196:78I-788.

16. Tyson KL, Bell Al, Cole JA, Busby SJW: Definition of the nitrite and nitrate response elements at the anaerobically inducible Escherichia coli nirB promoter: interactions between FNR and NarL. Mol Microbiol 1993, 7:15I-I57.

17. Tyson KL, Cole JA, Busby SJW: Nitrite and nitrate regulation at the promoters of two Escherichia coli operons encoding nitrite reductase: identification of common target heptamers from NarP- and NarL-dependent regulation. Mol Microbiol 1994, 1 3:1045-1055.

18. Wu HC, Tyson KL, Cole JA, Busby SJW: Regulation of the E. coli nir operon by two transcription factors: a new mechanism to account for co-dependence on two activators. Mol Microbiol 1998, 27:493-505.

19. Bell Al, Cole JA, Busby SJW: Molecular genetic analysis of an FNR-dependent anaerobically-inducible Escherichia coli promoter. Mol Microbiol 1990, 4: $1753-1763$.

20. Darwin AJ, Stewart V: Nitrate and nitrite regulation of the Fnrdependent aeg-46.5 promoter of Escherichia coli K-I 2 is mediated by competition between homologous response regulators (NarL and NarP) for a common DNA-binding site. J Mol Biol 1995, 25 I: I5-29.

21. Jacquelin DK, Filiberti A, Argarana CE, Barra JL: Pseudomonas aeruginosa MutL protein functions in Escherichia coli. Biochem J 2005, 388:879-887.

22. Georgellis D, Kwon O, Lin EC, Wong SM, Akerley BJ: Redox signal transduction by the ArcB sensor kinase of Haemophilus influenzae lacking the PAS domain. J Bacteriol 183:7206-72/2.

23. Kalman LV, Gunsalus RP: Identification of a second gene involved in global regulation of fumarate reductase and other nitrate-controlled genes for anaerobic respiration in Escherichia coli. J Bacteriol 1989, |7|:3810-3816.

24. Darwin AJ, Tyson KL, Busby SJW, Stewart V: Differential regulation by the homologous response regulators NarL and NarP of Escherichia coli K-I2 depends on DNA binding site arrangement. Mol Microbiol 1997, 25:583-595.

25. Cavicchioli R, Chiang RC, Kalman LV, Gunsalus RP: Role of the periplasmic domain of the Escherichia coli NarX sensor-transmitter protein in nitrate-dependent signal transduction and gene regulation. Mol Microbiol I996, 21:901-9II.

26. Chiang RC, Cavicchioli R, Gunsalus RP: "Locked-on" and "lockedoff" signal transduction mutations in the periplasmic domain of the Escherichia coli NarQ and NarX sensors affect nitrate- and nitrite-dependent regulation by NarL and NarP. Mol Microbiol 1997, 24:1049-1060.

27. Williams SB, Stewart V: Nitrate- and nitrite-sensing protein NarX of Escherichia coli K-I2: mutational analysis of the amino-terminal tail and first transmembrane segment. J Bacteriol 1997, 179:721-729.

28. Williams SB, Stewart V: Discrimination between structurally related ligands nitrate and nitrite controls autokinase activity of the NarX transmembrane signal transducer of Escherichia coli K-12. Mol Microbiol 1997, 26:911-925.

29. Bartolini E, Frigimelica E, Giovinazzi S, Galli G, Welsch JA, Granoff DM, Grandi G, Grifantini R: Role of FNR and FNR-regulated, sugar fermentation genes in Neisseria meningitidis infection. Mol Microbiol 2006, 60:963-72.

30. Wang $H$, Tseng C-P, Gunsalus RP: The napF and narG nitrate reductase operons in Escherichia coli are differentially expressed in response to submicromolar concentrations of nitrate but not nitrite. J Bacteriol 1999, I 81:5303-5308.

31. Snyder LA, Davies JK, Saunders NJ: Microarray genomotyping of key experimental strains of Neisseria gonorrhoeae reveals gene complement diversity and five new neisserial genes associated with Minimal Mobile Elements. BMC Genomics 2004, 5:23.

32. Snyder LA, Jarvis SA, Saunders NJ: Complete and variant forms of the 'gonococcal genetic island' in Neisseria meningitidis. Microbiol 2005, I 5 I:4005-40I3.

33. Marchler-Bauer A, Bryant SH: CD-Search: protein domain annotations on the fly. Nuc Acids Res 2004:W327-3I.

34. Kellogg DS Jr, Peacock WL, Deacon WE, Brown L, Pirkle Cl: Neisseria gonorrhoeae. I. Virulence genetically linked to clonal variation. A Bacteriol |963, 85: 1274-1279.

35. Snyder LA, Saunders NJ: The majority of genes in the pathogenic Neisseria species are present in non-pathogenic Neisseria lactamica, including those designated as 'virulence genes'. BMC Genomics 2006, 30: 128 .

36. Uzzau S, Figueroa-Bossi N, Rubino S, Bossi L: Epitope tagging of chromosomal genes in Salmonella. Proc Natl Acad Sci 200I, 98:15264-15269.

37. Mesa S, Ucurum Z, Hennecke H, Fischer HM: Transcription activation in vitro by the Bradyrhizobium japonicum regulatory protein FixK2. J Bacteriol 2005, 187:3329-38.

38. Grainger DC, Overton TW, Reppas N, Wade JT, Tamai E, Hobman JL, Constantinidou CC, Struhl K, Church G, Busby SJ: Genomic Studies with Escherichia coli MelR Protein: Applications of Chromatin Immunoprecipitation and Microarrays. J Bacteriol 2004, 186:6938-6943.

39. Aparicio O, Geisberg JV, Sekinger E, Yang A, Moqtaderi Z, Struhl K: Chromatin Immunoprecipitation for Determining the Association of Proteins with Specific Genomic Sequences In Vivo. Current Protocols in Molecular Biology 2005:21.3.1-21.3.33.

40. Datsenko KA, Wanner BL: One-step inactivation of chromosomal genes in Escherichia coli K-1 2 using PCR products. Proc Natl Acad Sci USA 2000, 97:6640-5.

4I. Liu JD, Parkinson JS: Genetics and sequence analysis of the pcnB locus, an Escherichia coli gene involved in plasmid copy number control. I Bacteriol 1989, I7 I: |254-6I.

42. Pope NR, Cole JA: Generation of a membrane potential by one of two independent pathways for nitrite reduction by Escherichia coli. J Gen Microbiol I982, I 28:319-322.

43. Silver LE, Clark VL: Construction of a translational lacZ fusion system to study gene regulation in Neisseria gonorrhoeae. Gene 1995, 166:101-104.

44. Macdonald $\mathrm{H}$, Cole JA: Molecular cloning and functional analysis of the cysG and nirB genes of Escherichia coli K-12, two closely linked genes required for NADH-dependent nitrite reductase activity. Mol Gen Genet 1985, 200:328-334. 